\title{
KNOWLEDGE-BASED SIGNAL PROCESSING FOR RADAR IDENTIFICATION
}

\author{
Jan Matuszewski \\ Institute of Radioelectronics, Faculty of Electronics, \\ Military University of Technology \\ 2 Gen. S. Kaliskiego St. \\ 00-908 Warsaw, POLAND \\ e-mail: jmatuszewski@wel.wat.edu.pl
}

\begin{abstract}
The paper presents some aspects of radar signals acquisition in the electronic intelligence (ELINT) system, the analysis of their parameters, feature extraction using linear Karhunen-Loeve transformation and applying knowledge-based techniques to the recognition of the intercepted signals. The process of final emitter identification is based on "the knowledge-based approach" which was implemented during the processing of constructing the database.
\end{abstract}

Keywords: radar identification, eigenvalue vectors, weighted coefficients, knowledge base.

\section{INTRODUCTION ${ }^{1}$}

The last local military conflicts have shown that a success on the modern battlefield depends on the following aspects:

- detecting electromagnetic emissions and finding the direction of incoming signals;

- gathering basic information about an enemy's situation and his capabilities;

- analyzing the emitter's characteristics such as technical parameters, operating role and geographic location;

- providing current and real-time information about hostile activities in the areas of interest and warning against threats;

- monitoring any changes of situation and target parameters;

- transmission of the most important data as soon as possible;

- providing control, commanding and supporting for the forces on the electronic battlefield.

The present electronic intelligence system (ELINT) must be able to fulfill these specific requirements. One of the most principal functions of ELINT system is gathering basic information from the entire electromagnetic spectrum and its analysis.

The appropriate emitter database (DB) is one of the most important elements in the present ELINT and electronic warfare support measures (ESM)

\footnotetext{
${ }^{1}$ The paper is financed from sources for science in the years 2007-2010 in a frame of ordered scientific project PBZMNiSW-DBO-04/I/2007.
}

system.

The process of designing the optimal structure of emitter database is a very complicated and sophisticated task. The main problem which appears during the process of designing the database is the difficulty in correct selection of radar parameters and calculating the specific signature for each type of radar (emitter) [1]. Specially important is utilization of a emitter DB in the process of identifying a detectable radar emissions [2].

A main function of ESM system is to threat detection and the area surveillance so as to determine the identity of surrounding emitters. This system must be able to recognize emitters from the pulse-by pulse measurements made by receiver in order to indicate the presence of known (friendly or hostile) radar, as well as to provide emitter tracking, threat assessment and platform identification. The ELINT/ESM data processing, with response time constantly decreasing, presents one of the most complex and time-domain critical problem for current technology [3].

The typical automatic ESM system consists of the following subsystems, Fig.1:

1) Signal receiver/sensor: provides, with very short acquisition times, long range detection of emitter radiations and measurements of their superior parameters.

2) Signal processor: allows, in a dense electromagnetic environment, a very accurate analysis of complex radar signals, sorting the input data into pulse chains (trains) with the aim of producing one pulse train for each received emitter 
(this process is called de-interleaving). This analysis allows to set up the signature of signal parameters in order to precisely identify and update the radar library (database).

3) Radar signal classifier allows parameters extraction and selection/reduction the data incoming to the classifier, which successively compares the specific signal parameters (signatures) with a library of known emitter characteristics (compiled through intelligence service) to determine the possible identities of detected signals.

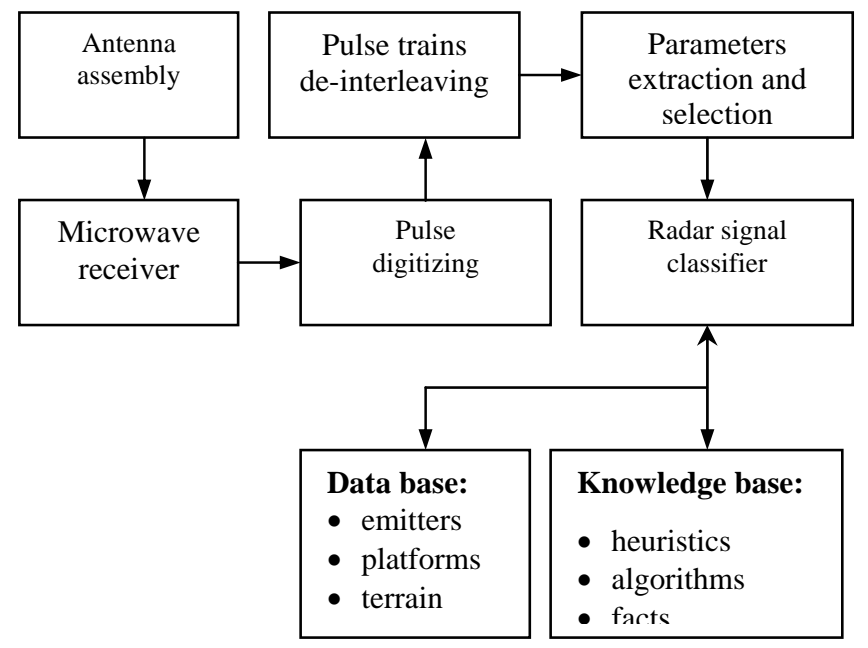

Fig. 1 - Structure of ESM signal processing scheme.

The modern ESM systems should be able to recognize emitters from the set of pulse measurements in order to provide surveillance, tracking and platform identification. They are required to react fast and with great accuracy in an extremely complex electromagnetic environment.

The electromagnetic environment is assumed to be complex, as there are signals from a number of different radars present, and signals from the same emitter are noisy or their parameters (features) are not measured with great accuracy.

In many cases, the radar may change one or several signal parameters in order to perform its task more efficiently. When pulses from unknown radar sources are being analyzed, they are usually separated into distinct groups, one group per radar or, more generally, per the radar's mode of operation. Since each radar has limited parameter ranges (e.g. transmit within a limited frequency band) and often identifiable characteristics, it is assumed that radar signals with similar characteristics originate with the same device.

The present ELINT system acquires the basic information from different spheres of activities, e.g. Signal Intelligence (SIGINT), Imagery Intelligence (IMINT), Human Intelligence (HUMINT), Measures and Signature Intelligence (MASINT). The characteristic of the present battlefield electromagnetic environment, the process of acquisition and transformation of the data show that measured basic parameters are not enough during the process of source identification.
In an emitter identification system, a received signal is analysed to extract some parameters that is necessary to estimate the type of radar. Conventional ESM systems measure the basic parameters of incoming radar signals. The typical radar parameters may be divided into two groups, primary and secondary. The primary parameters of radar signal, measured by the receiving subsystem are following: radio frequency (RF), time of arrival (TOA), pulse width (PW), angle of arrival (AOA) and amplitude (A). The main processor block derives the secondary radar parameters such as: pulse repetition interval (PRI), polarisation, variation of PRI (agility, jitter, stagger), variation (agility) of RF, scan type (ST), scan period (SP). By determining these parameters of a radar signal it is possible to identify the type, technical and operational role (assignment, designation), platform and the radar's user [3].

The collected data are sorted into groups considered to come from a single emitter and used to compute the time dependent parameters (pulse repetition frequency, antenna rotation period, etc.). Finally, the system matches the 'signal signature', composed by the average parameters from each group, with the characteristics of the identified emitters stored in the radar mode library data file. This action enables the system to identify or classify the incoming radar signals.

The success of modern electronic warfare depends on correctly designed database. The emitter database should include the patterns of radio-electronic devices, the technical characteristics of electromagnetic sources and the permanent possibility of data modification [2]. According to the available bibliography, the emitter database ought to possess approximately several thousand of emitters patterns. All information contained in database can be divided into logical, numerical, descriptive and graphic groups.

The designer of database should take into account measured and non-measured features, which means all accessible information on emission's source. The measured feature is a result of measurements and calculations. The non-measured feature can be expressed by the chain of words or logical expression. The classification of radar information is illustrated in Fig.2. All information classified in this way should be contained in radar signature.

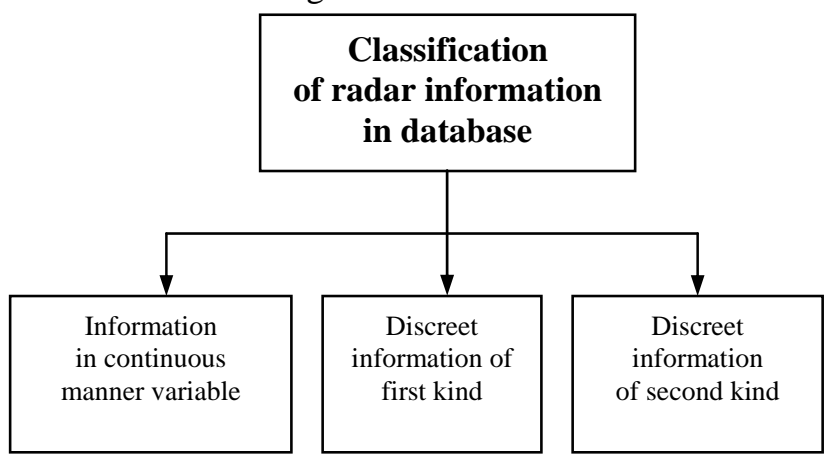

Fig. 2 - The classification of information included in database. 
The information in continuous manner variable can accept facultative values from selected section of real numbers' axis.

The discreet information group of first kind accepts discreet values such as $0 / 1$ (yes/not). These features are expressed by qualitative character of occurrence or lack of certain properties.

The third group of information referred to as "the discreet information of second kind" is characterized by not-derivative elements of an object's structure, expressed by words or characters chain.

\section{METHODS OF SIGNAL PROCESSING AND ANALYSIS OF ESM/ELINT DATA}

To ensure rapid system response in a dense and complex electromagnetic environment of a future conflict, the search, interception, analysis and identification functions have to be automated. The special software concerning measurements, processing, analysis and recognition of radar signals consists of the following parts [4]:

- control of radar signals measurement in ESM receiver,

- de-interleaving of the pulse trains which are then compared with an emitter library,

- analysis of intercepted signals to determine the set of radar parameters,

- calculation of a specific signal measurement signature to emitter database,

The first step in the process of examine the properties of radar signals is analysis of the measured data set and allows to determine:

- the type and range of changes of basic radar signal parameters, such as RF, PRI, PD;

- the type of intra-pulse modulation for complex signals and values of changes for signals with frequency modulation.

A few examples of intercepted signals from radars of different applications are presented in Figs. 3 to 8 . The analysis results are presented on two types of figures $[4,5]$. The first type of these figures (on the top) shows the measured values of A, PRI and RF for some number (tens to a few hundred) successively received pulses of intercepted radar signal. The second type of these figures (on the bottom) shows the envelope of a few pulses running successively, received on outputs AM and FM of a demodulator.

Fig. 3 illustrates the simple signals generated in two frequency channels by radar working in a diversity mode. The times of successively generated signals are shifted between them about the interval which is only a little different than their pulse width. The cycle change of PRI is six-positional.

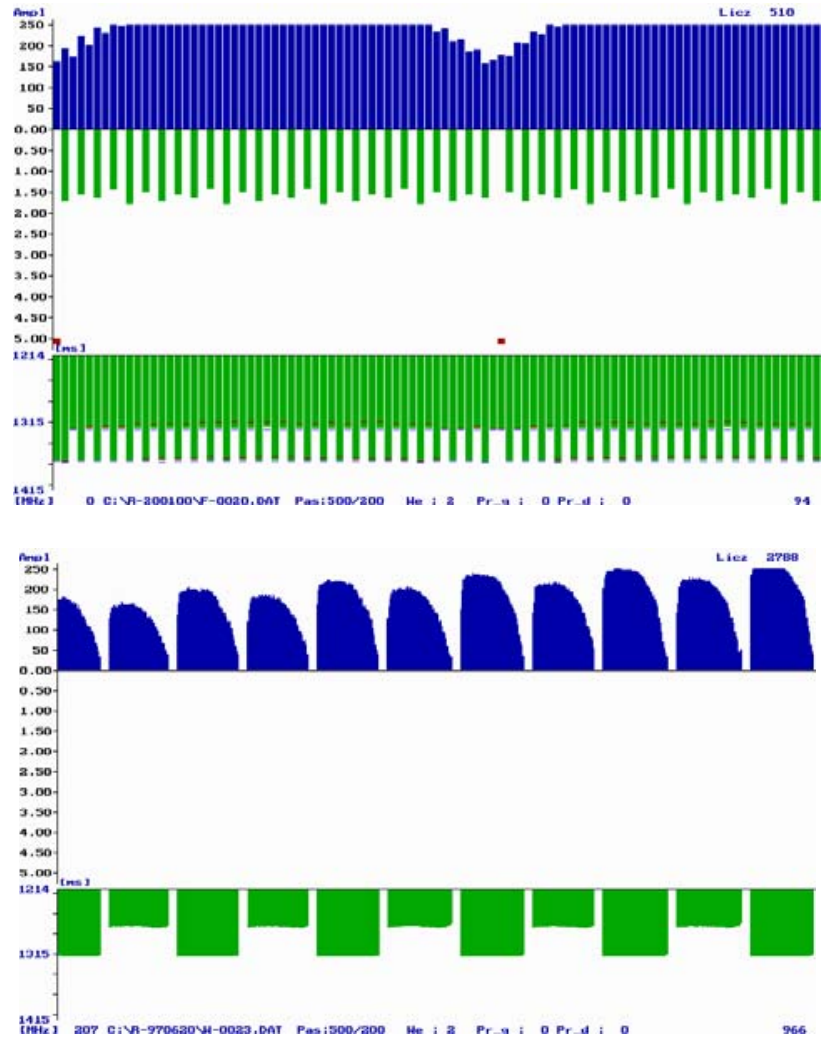

Fig. 3 - Land-based surveillance radar in band $L$.

Fig. 4 presents signals that are generated on two or more frequencies. Once these signals are generated simultaneously on a few frequencies while at another time it has been observed that the changes of RF take place from pulse to pulse in a fixed or random way. The simple signals are generated on many frequencies placed in $60 \mathrm{MHz}$ band and changes frequency take place from pulse to pulse in a random way.

Fig. 5 presents a complex signal with intra-pulse linear or non-linear frequency modulation generated by radar with two-beams antenna and assigned for searching targets at low and high altitudes.

Fig. 6 shows the complex signals with linear frequency modulation. Frequency of these signals has got six values. Change RF in $70 \mathrm{MHz}$ band follows step by step from group to group pulses (3 or 4 pulses) according with a determined rule. A signal has got a very sophisticated structure of PRI. In train pulses there are longer pulses. Most of them has got $t_{i}=8.2 \mu$ s although there are pulses with $t_{i}=32$ $\mu \mathrm{s}$ or $64 \mu \mathrm{s}$.

Fig. 7 shows signals generated by multifunction radars with sophisticated frequency and time structure. It genarates the complex signals in a band of $400 \mathrm{MHz}$ and frequencies change randomly. Additionally intra-pulse change frequency takes place by some value (step frequency). The frequency-time structure changes of emitted signals are very sophisticated and depend on radar working mode and distance to lightened target. 


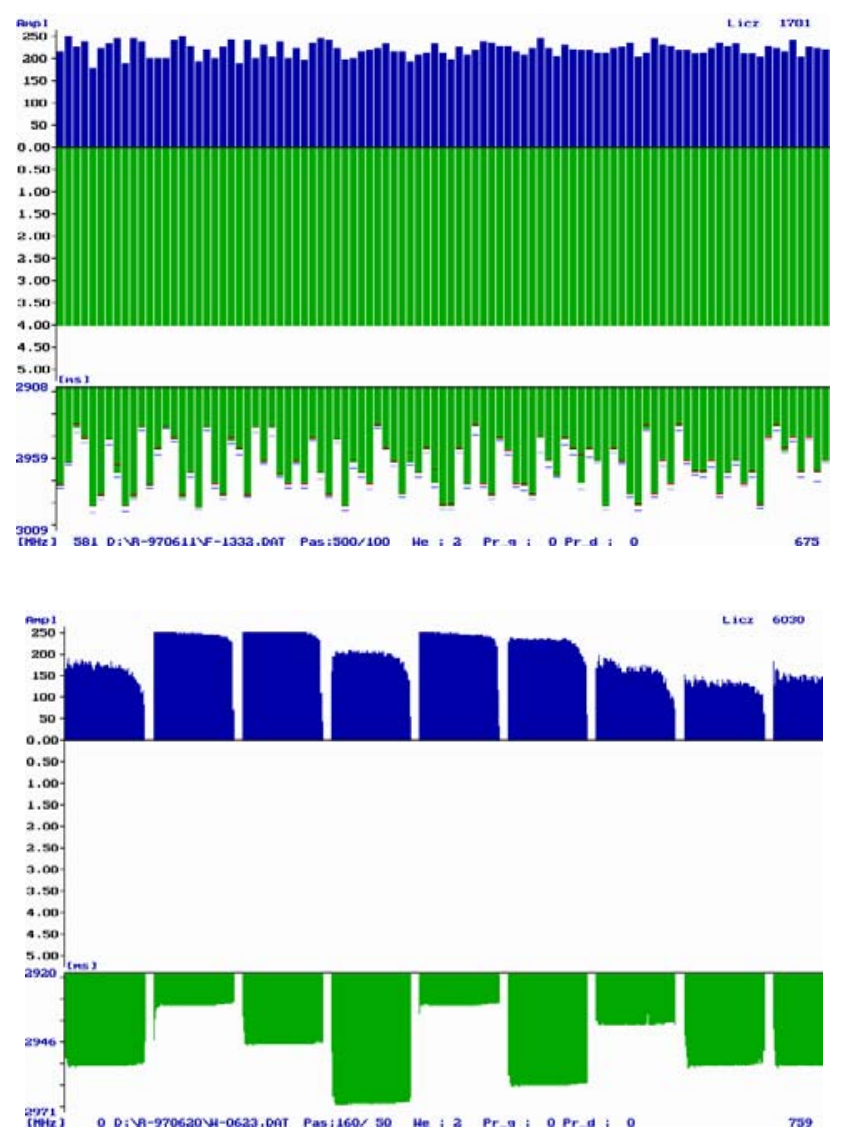

Fig. 4 - Land-based long-range 3D air defence radar in band $S$.

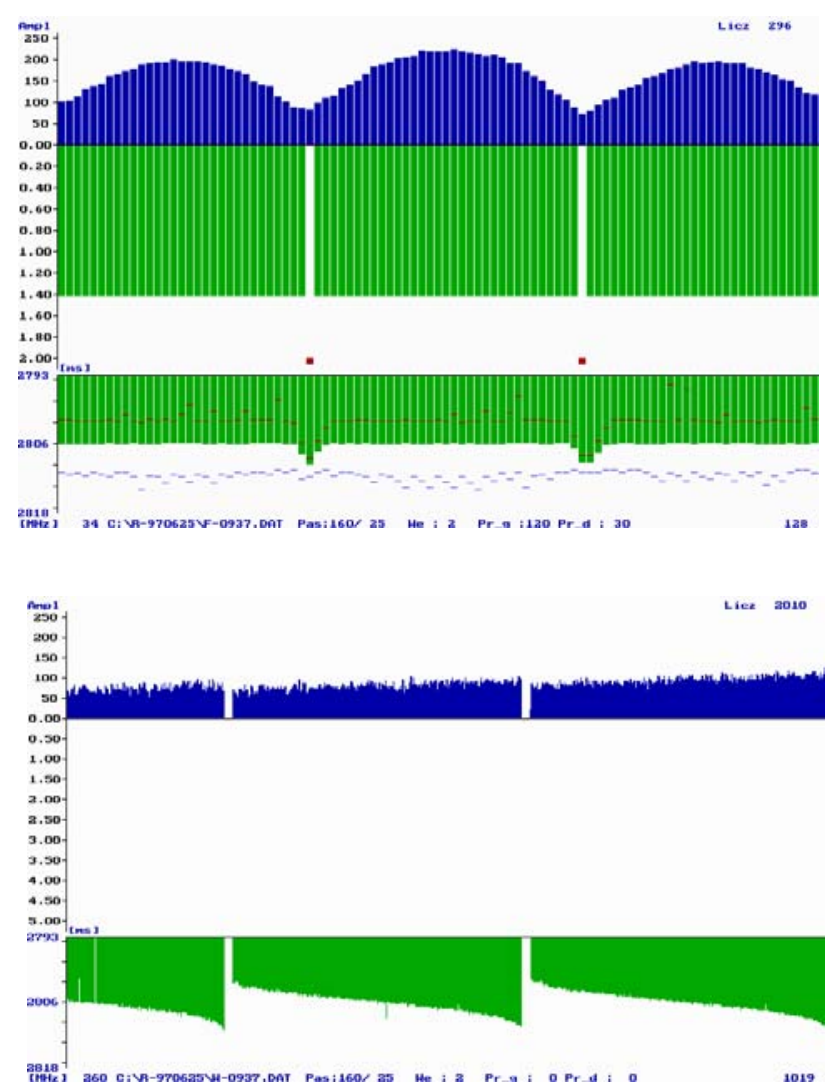

Fig. 5 - Naval air surveillance radar in band S.
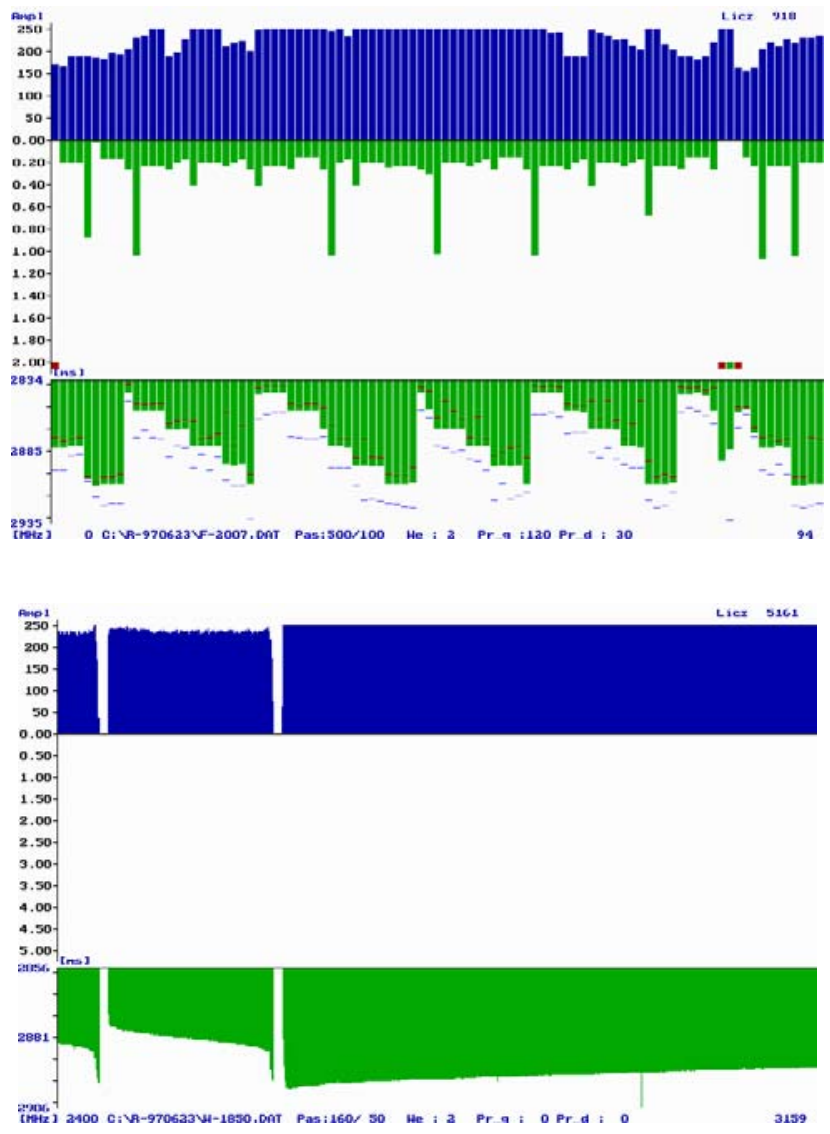

Fig.6 - Naval 3D surveillance and target indication radar for frigates in band $S$.
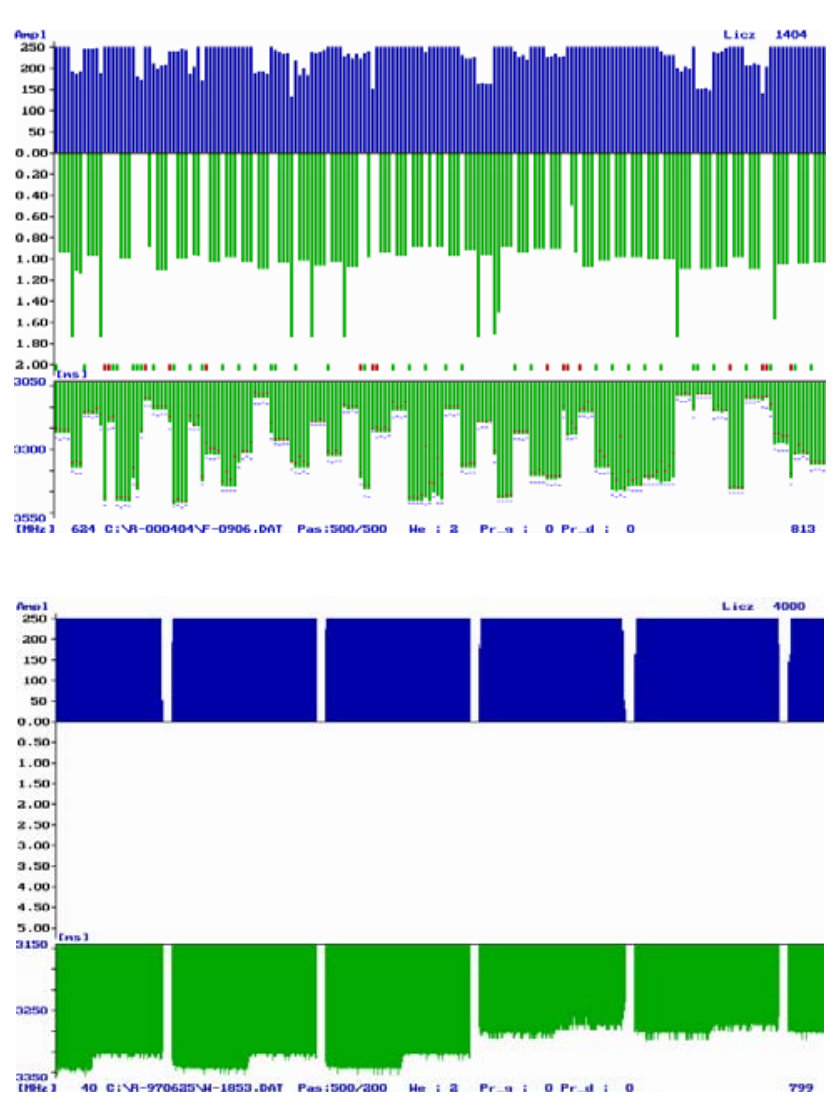

Fig. 7 - Naval multifunction array radar with electronic scanning in band $S$. 
Fig. 8 presents a complex signal with non-linear frequency modulation. Radar works on one of the 16 different frequencies from the band of $80 \mathrm{MHz}$. Depends on the mode of work, frequency changes after 4 or 6 pulses and its value is chosen in a random way. This kind of signal has got very sophisticated structure of PRI. In one of two modes of work 25 different values of PRI are generated while at the same time one of them is generated periodically after 4 pulses. In the second mode of work radar generates 4 different values PRI which appear in series of 5 or 6 sequence values.
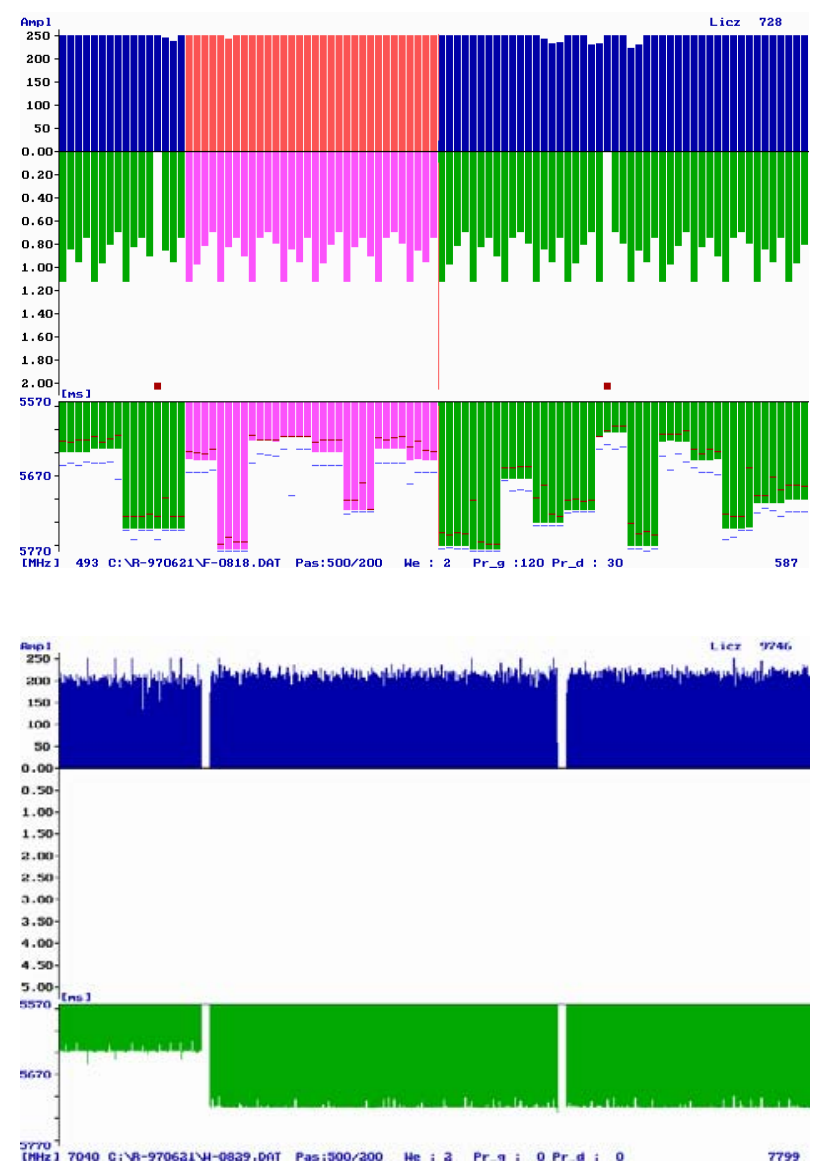

Fig. 8 - Land-based mobile 3D search radar in band C.

These shown above examples from different type of radars illustrate that radars of different applications have the specifical pictures which can help us in many times to recognize their applications.

\section{LINEAR KARHUNEN-LOEVE TRANSFORMATION}

In radar signal recognition a great deal of information collected by the receiver is processed in real time and computer must be used to analysis, feature extraction and recognition of the intercepted unknown signals. Most of the feature extraction methods presented in the literature are based on the linear Karhunen-Loeve transformation or eigenvector orthonormal expansion [6]. Since the eigenvectors can be ranked on the basis of corresponding them eigenvalues, that a subset of the best eigenvectors provides the optimal fitted subspace. Eigenvectors can be used in a such classifier, in which patterns are projected from multi-dimensional data onto one-, two- or maximum three dimensional spaces, so we can both observe the inherent structure of the data and design piecewise linear decision rule for signal classification. It is an optimal method in the mean square sense and it has a wide range of applications in reducing multi-dimensional data vectors by using only the first coefficients of transformation [6].

In further part of this paper the radar signal consisting of $N$ parametres will be assumed as a point in the $N$-dimensional Euclidean space and denoted as a vector $\boldsymbol{x}$ or in case of many samples by $\boldsymbol{x}_{k}$, where index $k$ denotes the number of the sample. In this procedure, the $N$-dimensional vectors $\boldsymbol{x}_{k}$ are transformed by multiplying by the eigenvectors $\boldsymbol{v}_{j}$ of estimated covariance matrix (within-class scatter) $\boldsymbol{A}$. The eigenvalues satisfy the following equation

$$
\boldsymbol{A} \boldsymbol{v}_{j}=\lambda_{j} \boldsymbol{v}_{j}, j=\overline{1, N}
$$

where: $\lambda_{j}$ - the eigenvalue corresponding to the $j$ th eigenvector.

For the real data measured parameters of radar signals, $\boldsymbol{A}$ is a real symmetric matrix and all the eigenvectors are orthogonal, i.e., $\boldsymbol{v}_{i}^{T} \boldsymbol{v}_{\boldsymbol{j}}=0$ for $i \neq j$ and the eigenvalues are all greater than or equal to zero. Hence the eigenvalues can be ordered such that

$$
\lambda_{1} \leq \lambda_{2} \leq, \ldots, \leq \lambda_{N}
$$

The matrix $\boldsymbol{V}=\left[\boldsymbol{v}_{1}, \boldsymbol{v}_{2}, \ldots, \boldsymbol{v}_{N}\right]^{T}$ is an orthogonal transformation such that $\boldsymbol{y}_{k}=\boldsymbol{V} \boldsymbol{x}_{x}$ and each $y_{j}=\boldsymbol{v}_{j}^{T} \boldsymbol{x}_{k}$ is the $j$ th feature in a new space for the input vector $\boldsymbol{x}_{x}$. The mean square error obtained by projecting all the data into a subspace spanned by only a subset of $K(K \leq N)$ eigenvectors can be minimized by choosing the eigenvectors corresponding to the $K$ smallest eigenvalues $\lambda_{i}$.

Although this transformation is optimal with respect to the given data, it is not necessarily optimal with respect to the selection of important features for separating pattern classes. When two pattern classes share similar important features, the corresponding eigenvalues are large and dominant. The important features for class 1 may be the least important features for class 2 and vice versa. Therefore, selection of the basis eigenvectors can be performed 
by taking $\boldsymbol{v}_{1}^{(1)}, \boldsymbol{v}_{2}^{(2)}, \ldots$ for class 1 and $\boldsymbol{v}_{N}^{(1)}, \boldsymbol{v}_{N-1}^{(2)}, \ldots$ for class 2 .

This technique does not extend to the general multiclass problem. However, the multiclass pattern classifier may be realized as a sequence of pairwise comparisons of likelihood functions. In this case each pair of classes may be examined using the expansion described above. Another way of solving this multiclass problem is to calculate at the beginning the mean covariance matrix $\boldsymbol{A}$ for all classes $L$ and next to compute the eigenvectors and eigenvalues for this matrix.

One of the advantages of calculating the eigenvalues is the possibility of using them to determine the "weighted coefficient" $\omega_{i}$ for radar signal parameters [6].

These coefficients may be determined for two conditions:

a) If

$$
\sum_{i=1}^{N} \omega_{i}=1
$$

then

$$
\omega_{j}=\frac{1}{\lambda_{j}}\left(\sum_{i=1}^{N} 1 / \lambda_{i}\right)^{-1}, \quad j=\overline{1, N}
$$

b) If

$$
\prod_{i=1}^{N} \omega_{i}=1
$$

then

$$
\omega_{j}=\frac{1}{\sqrt{\lambda_{j}}}\left(\prod_{i=1}^{N} \sqrt{\lambda_{i}}\right)^{1 / N}, j=\overline{1, N}
$$

The minimum long distance classifier calculates the weighted distance to each of $L$ known classes and classifies the samples $\boldsymbol{x}_{i}$ to this $j$ class, for which the following condition is fulfilled:

$$
d\left(x_{i}, \bar{x}_{l}\right)=\min _{j}\left(x_{i}-\bar{x}_{j}\right)^{T} V^{T} W^{T} W V\left(x_{i}-\bar{x}_{j}\right)
$$

where: $\bar{x}_{j}, \bar{x}_{I}$ - the mean vectors for class $j$ and $l$, $\boldsymbol{V}$ - the transform matrix consisting of eigenvectors $v_{i}, W$ - the diagonal matrix of weighted coefficients.

Calculations, which have been done on the sample consisting of radar signals from 125 classes, 4 features and 1500 data vectors, showed that using these weighted coefficients in minimum long distance classifier decreased the error probability of classification by about $10 \div 15$ percent [6].

\section{APPLYING THE KNOWLEDGE BASED APPROACH TO EMITTER IDENTIFICATION}

Most often information concerning the radar platforms known to be present, their locations, their intentions, their history of recent operational use, behaviour and their expected actions is not used. For the most part information available to the knowledge-based ESM systems takes the form of individual radar details, their platforms and relationships between them.

The most important characteristics, which are related to each other in a specific type of radar and permit to recognize its application, are the following [3]:

- the principal parameters of radar signal that correspond to the technical characteristics of the radiating set are: pulse width, carrier frequency, pulse shape, pulse repetition frequency, type of scan, beam width;

- carrier frequency is related to: antenna dimension, beam width, maximum range of radar;

- pulse width affects: range resolution and minimum range of radar;

- pulse repetition frequency limits the maximum unambiguous range of a pulse radar;

- type of scanning (circular, sector, conical, helical, spiral etc.) is related to the application of radar.

The knowledge base employs a declarative, rulebased representation of facts about the radar parameters [7]. Radar has been employed on the ground, in the air, on the sea and in space. Groundbased radar has been applied mainly to the detection and location of aircraft or space targets. Shipboard radar may observe other ships or aircrafts, or it may be used as a navigation aid to locate shorelines or buoys. Airborne radar maybe used to detect other aircrafts, ships, or land vehicles, or it may be used for storm avoidance and navigation. The nature of the vehicle that carries the radar and the environment in which it operates have a significant influence on its design [3].

The carrier frequency emitted by a radar constitute one of the fundamental information of intercept. Factors related to carrier frequency are: antenna dimensions, beam, width, maximum range, type of target to be detected. Low frequencies $(200 \div 3000 \mathrm{MHz})$ permit large ranges, but require large antenna systems unable to give narrow beams. High frequencies $(3 \div 20 \mathrm{GHz})$ permit smaller ranges, require small antennas with more capability to produce narrow beams and to give high angular 
resolution.

Pulse width (PW) affects the range resolution and the minimum range of a radar. The range resolution of a radar is the minimum detectable difference in range between two targets. If two or more targets are distant from each other less than minimum range, they are presented as only one target. The minimum range of a radar is the minimum distance from the radar that a target may be still appears on the radar indicator. Both range resolution and minimum range are given by:

$$
R_{\min }=\frac{c P W}{2}
$$

where: $R_{\min }$ - minimum range or range resolution, $C$ - velocity of electromagnetic waves propagation, $P W$ - pulse width.

The maximum range of a radar depends on the medium power emitted by the antenna. But the medium power, besides other factors, depends on the pulse duration, therefore the larger is the distance the radar can operate, the larger the pulse width must be.

Pulse repetition frequency (PRF) limits the maximum unambiguous range of a pulse radar. The maximum unambiguous range is the maximum distance between target and radar such that a pulse echo can return to the receiver before the next pulse is transmitted. Therefore the pulse repetition time must be long enough to permit a pulse echo to get the receiver before the next pulse is emitted.

The scan rate varies from 3 to 30 revolutions per minutes. Usually low rotation speeds are used for long range radars, since scan rate must be low enough in comparison with PRF in order to permit each scan.

Type of scanning (circular, sector, conical, helical, spiral, raster, etc.) is related to the application of radar.

The beam width affects on the degree of the angular resolution of a radar. Angular resolution is the ability of a radar to discriminate between two targets at the same range but at a different azimuth and/or elevation. Narrow beams are more easily produced at higher frequencies, while at the lower frequencies larger reflectors are needed to get the same gain. In other words, the dimension of the antenna (or reflector) can be produced as the carrier frequency of operation increases (with the same efficiency).

The knowledge base employs a declarative, rulebased representation of facts about the radar domain. Generally, a scheme of emitter identification may be based on rules or semantic networks. The simplest way of knowledge representation by rules is as follows [5, 7]:
IF fact $A$ is true AND fact $B$ is true THEN conclude $X$ ELSE conclude $Y$

e.g.

IF (pulse width is less than $0.5 \mu$ s) AND (type of scan is circular-sector) THEN (the radar of battlefield surveillance was detected);

IF (pulse width is less than $0.5 \mu$ s) THEN (detected battlefield surveillance radar with probability 0.9 ).

In the real electronic warfare systems such procedures are much more sophisticated. Searching for the best solution needs other more complicated strategies, Fig. 9.

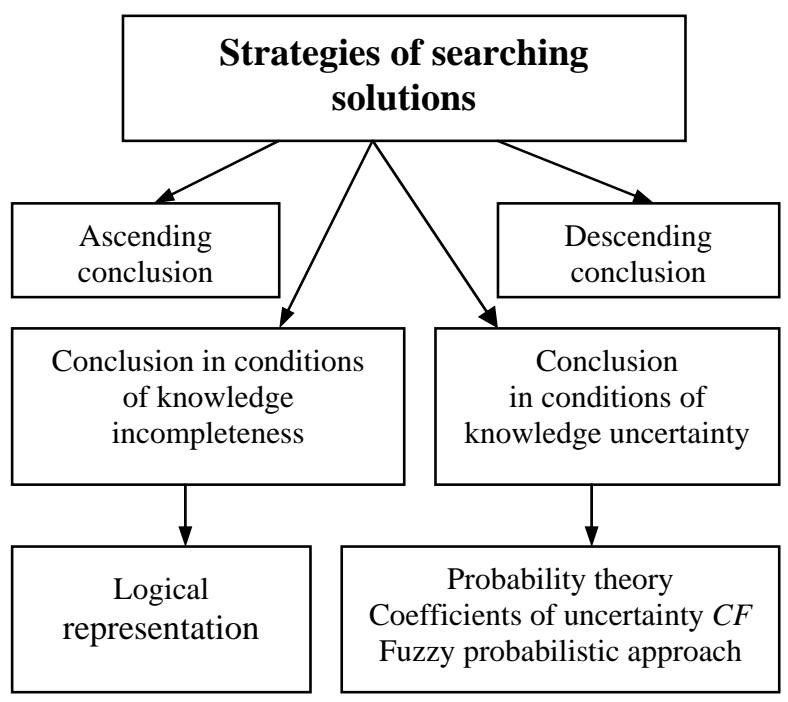

Fig. 9 - Strategies of searching solutions.

Knowledge presented in figure 9 by rules is a set of facts appropriately organized. If a set of such stated rules and facts obtain a true state, then the result of a final conclusion should be flashed to the operator. In order to make a decision in case of uncertainty, the probability theory or confidence factors (CF) may be used. The rules are activated in the moment when the values of this CF exceed the calculated thresholds, e.g.

\section{IF fact $(C F=0,3)$ THEN conclusion}

In a fuzzy probabilistic approach, the classical two-values function $f$ of membership of the object $\omega$ to set $A$, is replaced by a following 'fuzzy' function

$$
\begin{array}{r}
f(\omega)= \begin{cases}1, & \text { if } \omega \in A \\
0, & \text { if } \omega \notin A\end{cases} \\
f: A \rightarrow\{0,1\}
\end{array}
$$


In the description of a real situation the following concepts: 'nearly', 'enough', 'a little' which mirror the surrounding reality very well, are often used.

\section{CONCLUSION}

The capability of an ESM/ELINT system to correctly identify detectable radar emissions in a dense environment is a key to their application in modern command, communication and control system. The recording data and results of their analysis help us to extract some facts to constructing the knowledge base and design the expert systems in the last stage of radar identification.

The methods presented in this paper are particularly useful in such cases where set $L$ classes may be divided on subsets containing two or a few classes. In electromagnetic environment values of radar signal parameters often change. Every time in a such case we have to calculate the new, eigenvectors, eigenvalues and appropriate weighted coefficients.

The effective signal processing can be achieved by the special multiple-processor of high speed with a large memory for the radar parameters window addressing, radar signal identification, correlation and visualization on the monitors. Furthermore, the development of these techniques provides a powerful way ahead for their application to other related signal processing areas such as image processing and expert systems.

\section{REFERENCES}

[1] J. Dudczyk., J. Matuszewski, M. Wnuk. Applying the relational modelling and knowledge techniques to the emitter database design. Journal of Telecommunications and Information Technology. (1/2003), pp. 51-54.

[2] J. Dudczyk, M. Wnuk, J. Matuszewski. Applying the radiated emission to the specific emitter identification. Journal of Telecommunications and Information Technology. (2/2005), pp. 57-60.

[3] R. G. Wiley. Electronic Intelligence: The Analysis of Radar Signals. Second edition, Artech House, Boston \& London, 1993.
[4] J. Matuszewski, K. Banasiak, S. Wnuczek. Some aspects of radar signals acquisition, analysis and recognition. Proceeding of "Sensors \& Electronics Technology Panel". SET Symposium, Warsaw, Poland, 23-25 April 2001, RTO-MP-070 AC/323 (SET-047) TP/17, pp. 44-1 - 44-12.

[5] J. Matuszewski, A. Kawalec. Knowledge-based Signal Processing for Radar Identification. Proceedings of the " $9^{\text {th }}$ International Conference on Modern Problems of Radio Engineering, Telecommunications and Computer Science TCSET'2008”. Lviv-Slavsko, Ukraine, 19-23 February 2008, pp. 302-305.

[6] J. Matuszewski. Appplication of Eigenvalue Analysis and Discriminant Vectors in Radar Signal Recognition, Proceedings of "Nove smery $v$ spracovani signalov III”, Liptovsky Mikulas, Slovakia, 29-31 May 1996, pp. 148151.

[7] J. Matuszewski, L. Paradowski. The Knowledge Based Approach for Emitter Identification. Conference Proceedings of " $12^{\text {th }}$ International Conference on Microwaves \& Radar, MIKON'98”, Kraków, Poland, 20-22 May 1998, vol. 3, pp. 810-814.

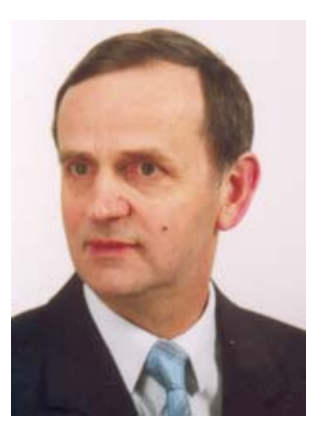

Jan Matuszewski was born in Cekcyn, Poland, in 1948. He received his M.Sc. degree in cybernetics engineering in 1972 and Ph.D degree in telecommunication in 1984 from the Military University of Technology (MUT), Warsaw, Poland

Dr Matuszewski works as an Assistant Professor at the MUT, Faculty of Electronics, Institute of Radioelectronics and his research interests include electronic warfare, radar signal processing, emitter classification and identification, designing emitter data base, application of expert systems and neural network to radar signal recognition. He is the author of 80 scientific publications. 